\title{
One-Year Surveillance of Surgical Site Infections at a Tertiary Care Surgical Center in Egypt
}

\author{
Mohammed Elshaer ${ }^{*}{ }^{(\mathbb{D}}$, Eman El-Sayed ${ }^{1}$ and Noha El-Mashad ${ }^{1}$ \\ ${ }^{1}$ Clinical Pathology Department, Mansoura Faculty of Medicine, Mansoura, Egypt
}

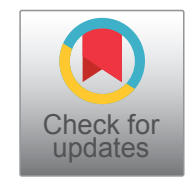

\begin{abstract}
Background: Surgical site infections are the commonest type of healthcare-associated infections in patients undergoing surgery, with higher overall cost and prolonged duration of hospital stay, although they are largely preventable.

Objectives: The aim of the study was to determine the incidence of surgical site infections at Mansoura Gastrointestinal Surgical Center amongst patients undergoing colonic, pancreatic and rectal surgery, also to determine bacteriological profile and antimicrobial susceptibility patterns of obtained isolates.
\end{abstract}

Methods: Between May 2019 and April 2020, swabs were collected from infected surgical wounds. Microbial identification and antimicrobial susceptibility were done by Vitek 2 system. All the data were collected using national electronic data surveillance system (NEDSS) developed by CDC and implemented by an active infection control team inside the hospital.

Results: The mean incidence of surgical site infections in our facility was $26.1 \%$. SSI have occurred more in colon surgery $37.3 \%$, than in pancreatic $33.7 \%$ and gastric surgery $23.6 \%$ with a high overall incidence of Organ/Space SSI than superficial and deep SSI.E. coli was the commonest organism (40.6\%), followed by Klebsiella (28.1\%) and Pseudomonas (10.9\%). An antibiogram was defined for all bacterial isolates obtained from surgical wounds to be considered in empirical antibiotic selection.

Conclusion: The incidence of SSI in patients undergoing colonic, pancreatic, and gastric surgery in our study was within values generally reported in other studies. Although SSIs cannot be completely eliminated, it can be reduced to minimal by proper adherence to infection control guidelines together with the implementation of an adequate antibiotic policy.

\section{Keywords}

Surgical wound infection, Infection control, Incidence, Centers for disease control and prevention, Anti-Bacterial agents

\section{Introduction}

Surgical site infections (SSI) are the commonest type of healthcare-associated infections in patients undergoing surgery, with higher overall cost and prolonged duration of hospital stay, although they are largely preventable [1].

The definition criteria for SSI were proposed by the Center for Disease Control and Prevention (CDC) to standardize data collection for the National electronic data Surveillance system (NEDSS). Accordingly, SSIs were classified into incisional SSIs, which can be superficial or deep, and organ/space SSIs, which affect the rest of the body other than the body wall layers [2].

Despite the advance in infection control practices such as adequate ventilation in operation room, barrier precautions and aseptic techniques, Infection at the surgical site remains as major source of morbidity and mortality following surgical procedures [3]. Continuous surveillance of SSI with periodic feedback of data to surgeons is considered an important preventive strategy to reduce the risk of SSI [4].
Gastrointestinal Surgical Center of Mansoura is a 170bed teaching hospital serving as a tertiary care hospital. An infection control team with 2 full-time nurses was maintained in our hospital. The team also acts as an advisory body to the medical staff, analyzing the epidemiology of the infections, improving the appropriate use of antimicrobials and providing adequate training for healthcare workers.

*Corresponding author: Dr. Mohammed Youssef Elshaer, Department of Clinical Pathology, Faculty of Medicine, Mansoura University Post, Mansoura, Egypt, 35516, Tel: +20102048-0202

Accepted: December 09, 2021

Published online: December 11, 2021

Citation: Elshaer M, El-Sayed E, El-Mashad N (2021) One-Year Surveillance of Surgical Site Infections at a Tertiary Care Surgical Center in Egypt. Arch Community Med 4(1):30-33 
Our study aimed to determine the incidence of surgical site infections at Mansoura Gastrointestinal Surgical Centeramongst patients undergoing colonic, pancreatic, and rectal surgery, also to determine the bacteriological profile and antimicrobial susceptibility patterns of obtained isolates.

\section{Ethics statement}

The study was conducted after approval of the Institutional research board of Mansoura Faculty of Medicine, Egypt.

\section{Type of the study}

A one-year descriptive database-based cohort study was carried out between May 2019 and April 2020 at Mansoura Gastrointestinal Surgical Center.

\section{Inclusion criteria}

Surgical site infections were defined according to the CDC guidelines as infections that occurs within 30 days following surgery and classified into superficial, deep incisional or organ/space infection with at least one of the following.

1. Purulent discharge from the wound site.

2. Organisms isolated from fluid or tissue cultures.

3. At least one of the signs or symptoms of infection: pain or tenderness, localized swelling, redness, or heat

\section{Diagnosis of SSI by attending surgeon.}

Monitoring for SSI was performed following surgical operation till discharge, including any readmission or postdischarge examination in outpatient clinic till the $30^{\text {th }}$ postoperative day.

\section{Exclusion criteria}

1- Infections occurring more than 30 days after surgery.

2- Patients with incomplete medical records.

\section{Data collection}

All the data were obtained by national electronic data surveillance system (NEDSS) developed by CDC and implemented by an active infection control team operating in the hospital using Personal digital assistants (PDAs) to facilitate data entry.

Hospital surveillance coordinators were responsible for screening patients in collaboration with treating physicians for any signs or symptoms suggestive of surgical site infection. They also reviewed laboratory and radiologic reports. If an SSI was suspected, then information entered the PDA was run against an SSI case definition stored in the PDA to evaluate whether case criteria were met or not.PDA data were uploaded to the CDC weekly for review and analysis.

\section{Methods}

\section{Sampling}

Swabs from postoperative infected surgical wound were collected under aseptic techniques. All samples were processed at Mansoura Gastrointestinal Surgical Center Laboratory, Egypt.
Samples were inoculated onto blood agar, chocolate, and MacConkey agar plates (Oxoid, United Kingdom) incubated at $37^{\circ} \mathrm{C}$ aerobically in MacConkey and blood agar. Plates were examined after 24 and 48 hours. Anaerobic culture is not a routine work in our laboratory and performed only by request, therefore the results were excluded.

\section{Microbial identification}

All bacterial isolates were identified to genus level by Vitek2 system (Biomérieux, France). Bacterial colonies were suspended in $3 \mathrm{ml}$ of sterile saline to the equivalent of a 0.5 McFarland turbidity using DensiChek plus (Biomérieux, France).

Vitek 2 Identification cards were filled with standardized bacterial suspension, and incubated at $35.5{ }^{\circ} \mathrm{C}$, and optical density was measured by the device every 15 minutes. Final identification results were obtained within 8-12 hours.

\section{Antibiotic susceptibility testing}

Antimicrobial susceptibility testing was done by disk diffusion using Vitek2 system (Biomérieux, France) using GP67 cards for gram positive isolates and GN-73 cards for gramnegative isolates.

\section{Statistical analysis}

All results were collected, tabulated, and statistically analyzed using SPSS Statistics version $\mathbf{2 1 . 0}$

\section{Results}

A total of 245 patients operated for colonic, pancreatic and gastric surgery at Mansoura Gastrointestinal Surgical Center between May 2019 and April 2020. The median patient age was 53 years. Most samples were collected from male patients (53.1\%; 46.9\% from female) (Table 1 ).

Regarding the distribution of pathogens associated with surgical site infections in our study; E. coli was the commonest organism (40.6\%), followed by Klebsiella (28.1\%), Pseudomonas (10.9\%), Enterococcus fecalis, Staph. aureus (6.3\%) for each, Coagulase-negative staphylococci (CONS) (4.7\%) and Acinetobacter baumanii (3.1\%) as illustrated in Figure 1.

The mean incidence of SSI in our facility was $26.1 \%$. SSI have occurred more in colon surgery $37.3 \%$, than in pancreatic $33.7 \%$ and gastric surgery $23.6 \%$ with a high overall incidence of Organ/Space SSI than superficial and deep SSI (Table 2).

A detailed antibiogram including all isolated grampositive and gram-negative bacteria is illustrated in Table 3. E coli and klebsiella isolates showed the highest sensitivity to meropenem $88 \%$ and $79.5 \%$ respectively in comparison very low sensitivity was noted with ampicillin $47 \%$ and $21.3 \%$ respectively. Pseudomonas isolates showed a very good sensitivity to amikacin $91 \%$ and gentamicin $84 \%$ with a high overall resistance against cephalosporins. Acinetobacter baumanii was the most resistant bacterial species observed in our study.

Tetracycline and linezolid were the most effective antibiotics against gram positive bacteria with almost $100 \%$ 
Citation: Elshaer M, El-Sayed E, El-Mashad N (2021) One-Year Surveillance of Surgical Site Infections at a Tertiary Care Surgical Center in Egypt. Arch Community Med 4(1):30-33

Table 1: Characteristics of patients with surgical site infections.

\begin{tabular}{|c|c|c|c|c|c|c|c|c|}
\hline \multicolumn{4}{|c|}{ Gender } & Median Age & $\begin{array}{c}\text { Median duration } \\
\text { of detection of } \\
\text { SSI (days) }\end{array}$ & $\begin{array}{c}\text { Median length } \\
\text { of pre-operative } \\
\text { stay (days) }\end{array}$ & $\begin{array}{c}\text { Median length of } \\
\text { post-operative } \\
\text { stay (days) }\end{array}$ & $\begin{array}{c}\text { Mortality rate } \\
\text { (\%) }\end{array}$ \\
\hline Mo. & \multicolumn{2}{|c|}{ Female } & (dars) & & \\
\hline 34 & 53.1 & 30 & 46.9 & 53.0 & 4.0 & 2.0 & 9.5 & 6.3 \\
\hline
\end{tabular}

Duration of detection of SSI: The time interval between the day of operation and the first appearance of sign or symptom of SSI.

Length of pre-operative stay: The time interval between day of hospital admission and day of operation.

Length of post-operative stay: The time interval between day of operation and day of hospital discharge.

Table 2: Incidence of surgical site infections by operation.

\begin{tabular}{|c|c|c|c|c|c|c|c|c|c|}
\hline \multirow{3}{*}{ Operation } & \multirow{3}{*}{$\begin{array}{c}\text { No. of } \\
\text { Operations }\end{array}$} & \multirow{3}{*}{ No. of SSIs } & \multirow{3}{*}{$\begin{array}{c}\text { SSI } \\
\text { Incidence }\end{array}$} & \multicolumn{6}{|c|}{ Type of Surgical Site Infections } \\
\hline & & & & \multicolumn{2}{|c|}{ Superficial SSIs } & \multicolumn{2}{|c|}{ Deep SSIs } & \multicolumn{2}{|c|}{ Organ/Space SSIs } \\
\hline & & & & No & $\%$. & No & $\%$. & No & $\%$. \\
\hline Pancreatic surgery & 86 & 29 & 33.7 & 9 & 10.5 & 0 & 0 & 20 & 23.3 \\
\hline Colon surgery & 59 & 22 & 37.3 & 10 & 16.9 & 0 & 0 & 12 & 20.3 \\
\hline Gastric surgery & 55 & 13 & 23.6 & 4 & 7.3 & 4 & 7.3 & 5 & 9.1 \\
\hline Total & 245 & 64 & 26.1 & 23 & 9.4 & 4 & 1.6 & 37 & 15.1 \\
\hline
\end{tabular}

Table 3: Antibiogram of bacterial isolates from Surgical wound infections.

\begin{tabular}{|l|c|c|c|c|c|c|c|}
\hline Selected antibiotic & E-coli & Klebsiella & Pseudomonas & $\begin{array}{c}\text { Acinetobacter } \\
\text { baumanii }\end{array}$ & S. aureus & CoNS & Enterococcus \\
fecalis
\end{tabular}

- Not all isolates tested against every antibiotic listed

- CONS: coagulase negative staphylococci

- Vitek2 system includes an advanced expert system (AES) that analyzes MIC patterns and detects phenotypes for most organisms tested. The AES also deduces susceptibility to drugs not tested based on antimicrobial phenotype of the strain

sensitivity whereas ampicillin and erythromycin were the least effective antimicrobials.

\section{Discussion}

Although there has been major progress in surgical techniques and better understanding of the pathogenesis of wound infection, management of SSIs is still considered a major problem in health care settings. In our study, the overall rate of SSI was $26.1 \%$. SSIs rates have been reported globally to range from $2.5 \%$ to $41.9 \%$ with a significantly higher incidence in low-income countries [5,6].

Most isolates were obtained from male patients $(53.1 \%$; $46.9 \%$ from female), however, it has been known that sex is not predeterminant of the risk of SSI. The incidence of SSI can vary from one surgeon to another as the skill and experience of the surgeon directly affects the condition of surgical site and therefore its resistance to infection also by the adherence of the medical staff to infection control guidelines implemented in the facility [7].

The duration of the hospital stay is a major risk factor for SSI as the longer the duration of hospital stay, the higher the risk of acquiring infection [8]. Moreover, the degree of bacterial contamination is fundamental to the risk of SSI. In our study, a $37.3 \%$ rate for colonic SSI was obtained. We hypothesized that the procedures of anastomosis were 
associated with contamination of the surgical site, because of the heavy bacterial load of the colon and rectum.

Gram-negative bacteria were the predominant pathogens in our study. This could be caused by the patient's endogenous flora. Similar observations have been reported in various studies by [9-11], whereas Pseudomonas aeruginosa was the most frequent isolate in studies by $[12,13]$.

Antibiotic susceptibility testing demonstrated a variable degree of resistance in different bacterial species which can be explained by their rational use of empirical antimicrobials as a prophylaxis and treatment in some patients might have contributed to observed resistance.

Acinetobacter baumanii was the most resistant bacterial species observed in our study. Acinetobacter species can accumulate multiple antibiotic resistance genes, leading to the development of multidrug-resistant or extensively drugresistant strains [14].

The present study was limited by its retrospective design; however, the strength of the study can be explained by its local nature, which reflects the magnitude of the problem of SSI in a tertiary surgical center in Egypt.

\section{Conclusions}

The incidence of SSI in patients undergoing colonic, pancreatic and gastric surgery in our study was within values generally reported in other studies. Although SSIs cannot be completely eliminated, it can be reduced to minimal by proper adherence to infection control guidelines together with the implementation of an appropriate antibiotic policy. The choice of antibiotics should follow the hospital policy whenever possible. If alternatives are chosen, the clinician must document the reason in the case records. The need for antimicrobial therapy should be reviewed daily.

\section{Informed Consent}

Verbal and written consent was obtained from all patients enrolled in the study.

\section{Funding/Support}

No funding or grant was obtained for this research.

\section{Conflict of Interests}

There were no conflicts of interest.

\section{References}

1. Haque M, Sartelli M, McKimm J, et al. (2018) Health care-associated infections-An overview. Infect drug resist 11: 2321-2333.

2. Horan TC, Andrus M, Dudeck MA (2008) CDC/NHSN surveillance definition of health care-associated infection and criteria for specific types of infections in the acute care setting. Am $\mathrm{j}$ infect control 36: 309-332.

3. Bratzler DW, Dellinger EP, Olsen KM, et al. (2013) Clinical practice guidelines for antimicrobial prophylaxis in surgery. Surg infect 14: 73-156.

4. Goyal R, Sandhu HPS, Kumar A, et al. (2015) Surveillance method for surgical site infection. Indian Journal of Pharmacy Practice 8: 54-60.

5. Organization WH (2011) Report on the burden of endemic health care-associated infection worldwide.

6. Horan TC, Andrus M, Dudeck MA (2008) CDC/NHSN surveillance definition of health care-associated infection and criteria for specific types of infections in the acute care setting. Am J Infect control 36: 309-332.

7. Cheng $\mathrm{H}$, Chen BP-H, Soleas IM, et al. (2017) Prolonged operative duration increases risk of surgical site infections: A systematic review. Surg infect 18: 722-735.

8. Carvalho RL, Campos CC, Franco LM, et al. (2017) Incidence and risk factors for surgical site infection in general surgeries. Rev Lat Am Enfermagem 25.

9. Chakraborty SP, KarMahapatra S, Bal M, et al. (2011) Isolation and identification of vancomycin resistant Staphylococcus aureus from post operative pus sample. Al Ameen J Med Sci 4: 152-168.

10. Ahmed MI (2012) Prevalence of nosocomial wound infection among postoperative patients and antibiotics patterns at teaching hospital in Sudan. N Am J Med Sci 4: 29-34.

11. Mulu W, Kibru G, Beyene G, et al. (2012) Postoperative nosocomial infections and antimicrobial resistance pattern of bacteria isolates among patients admitted at FelegeHiwot Referral Hospital, Bahirdar, Ethiopia. Ethiop J Health Sci 22: 7-18.

12. Masaadeh HA, Jaran AS (2009) Incident of Pseudomonas aeruginosa in post-operative wound infection. Am J Infect Dis 5: 1-6.

13. Sohn AH, Parvez FM, Vu T, et al. (2002) Prevalence of surgicalsite infections and patterns of antimicrobial use in a large tertiary-care hospital in Ho Chi Minh City, Vietnam. Infect Control Hosp Epidemiol 23: 382-387.

14. Lee CR, Lee JH, Park M, et al. (2017) Biology of acinetobacter baumannii: Pathogenesis, antibiotic resistance mechanisms, and prospective treatment options. Front Cell Infect Microbiol 7: 55.

DOI: $10.36959 / 547 / 647$

Copyright: (c) 2021 Elshaer M, et al. This is an open-access article distributed under the terms of the Creative Commons Attribution License, which permits unrestricted use, distribution, and reproduction in any medium, provided the original author and source are credited. 